\title{
L'ATTUALE DEFORMAZIONE DELLA CROSTA TERRESTRE $(*)$
}

\author{
Silvio Polli
}

Premesse. - In precedenti lavori, indicati nella bibliografia, ̀̀ stato messo in evidenza un procedimento che consente di misurare $i$ lenti movimenti verticali della superficie terrestre indipendentemente dalla variazione del livello medio marino e dagli spostamenti verticali costieri. 11 metodo dà pertanto misure che hanno un grado di assolutismo superiore a quelle che si ottengono prendendo come superfici di riferimento piani passanti per caposaldi della costa oppure livelli medi marini.

Esso si basa sulla determinazione della differenza fra le velocità del movimento verticale medio di tutti i mari e quello di una data stazione mareografica. Questa differenza dà lo spostamento verticale assoluto (in senso largo) del caposaldo mareografico nell'intervallo di tempo considerato. Quale applicazione di questo procedimento sono stati calcolati gli spostamenti verticali attuali dei caposaldi costieri per tutte le stazioni mareografiche di tutti i mari. Il calcolo è stato eseguito per periodi decennali nell'intervallo 1871-1940. Presentiamo nella tabella allegata un estratto dei valori riguardanti il cinquantennio 1891-1910.

$1 l$ materiale. - Sono state prese in considerazione tutte le stazioni mareografiche che disponevano di regolari serie di registrazioni per almeno un periodo continuo di 10 anni compreso nell'intervallo 1891-1940. Nel caso di due o più siti vicini e con registrazioni simili si scelse quello più regolare e significativo. Risultarono così selezionate 110 stazioni distribuite lungo tutti i mari e comprese tra i $64^{\circ}$ di latitudine Nord (Rönnskär, Finlandia) ed i $48^{\circ}$ di latitudine Sud (Deseado, Argentina). Molto scarse sono le stazioni nell'Africa, specialmente meridionale, e nell'America del Sud. Inutilizzabili al nostro scopo sono le brevi serie mareografiche eseguite dalle diverse spedizioni

(*) Comunicazione presentata al Convegno dell'Associazione Geofisica Italiana, tenuto a Roma il 17-18 giugno 1953. 


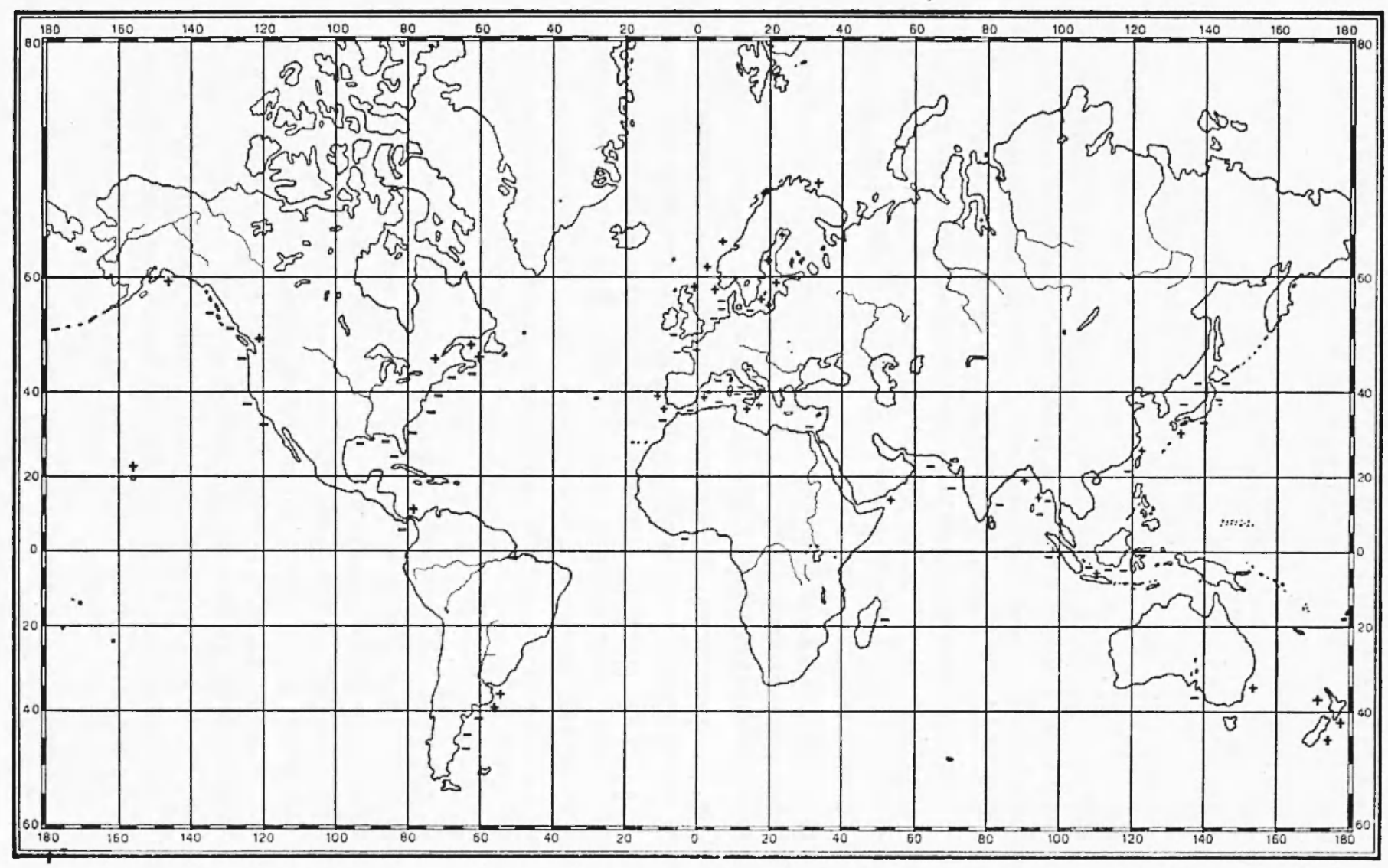


polari a latitudini superiori (in valore assoluto) a quelle sopraindicate. $\mathrm{Si}$ vedrà più avanti quanto preziosi potrebbero essere $\mathrm{i}$ dati di queste zone. Per evidenti ragioni di calcolo dei valori medi è necessario disporre di una distribuzione uniforme delle stazioni mareografiche lungo le coste di tutti i mari. Pertanto le stazioni vicine furono raggruppate secondo zone costiere o secondo mari, attribuendo ad ogni zona il valore medio di quelli corrispondenti alle singole località. I valori medi degli spostamenti verticali delle zone considerate, in $\mathrm{cm}$ per decennio, figurano nella stessa tabella allegata.

$1 l$ procedimento di ricerca. - Segnando su di un globo terrestre o su di una sua rappresentazione piana con un segno - le zone in fase di abbassamento e con un segno + quelle in fase di sollevamento, si vede che le prime sono più frequenti lungo una larga fascia equatoriale compresa circa fra i $45^{\circ}$ di latitudine Nord e i $45^{\circ}$ di latitudine Sud, mentre le seconde, cioè $i$ sollevamenti, sono più numerosi fuori di tale fascia, cioc̀ nelle due calotte polari situate sopra e sotto i $45^{\prime \prime}$ di latitudine Nord, rispettivamente Sud.

Questa visione qualitativa è confermata anche quantitativamente dai sewuenti calcoli numerici. Si determini, partendo dai dati della tabella, la media aritmetica dei valori degli spostamenti (considerati con il loro segno algebricol di tutte le zone comprese nelle due calotte polari situate a Nord e a Sud delle latitudini di $45^{\circ} \mathrm{N}$ e di $45^{\circ} \mathrm{S}$. Si olterrà il valore $+0.3 \mathrm{~mm} /$ decennio, corrispondente pertanto ad un sollevamento generale delle terre situate nelle due calotte polari.

Determinando invece, in modo analogo, lo spostamento verticale medio di tutte le regioni costiere comprese tra i paralleli di $45^{\circ}$ Sud e Nord, cioè situate nella fascia equatoriale, si arrà il valore $-0,2$ mm deconnio, corrispondente ad un abbassamento generale delle zone continentali che si trovano in questa fascia.

Ripetendo gli stessi calcoli per le zone equaloriali comprese tra le latitudini di $=55^{\circ},+50^{\circ}, 40^{\circ}, 35^{\circ}, \therefore 30^{\circ}$, e per le corrispondenti calotte complementari si otlengono i valori della seguente tabelliua:

Spostamonti verticali in $\mathrm{mm}$ decennio

$(+$ innalzamento, - abbassamento $)$

\begin{tabular}{|l|c|c|c|c|c|c|}
\hline \multicolumn{1}{|c|}{ Zona } & \multicolumn{5}{|c|}{ Iatitudini limiti tra la fascia equatoriale e le calotte polari } \\
& $-55^{\circ}$ & $\div 50^{\circ}$ & $\pm 15^{\circ}$ & $\pm 10^{\circ}$ & $\pm 35^{\circ}$ & $\pm 30^{\circ}$ \\
\hline Polare & $-1,70$ & -0.51 & $+0,32$ & $+0,12$ & $+0,09$ & $+0,09$ \\
Equatoriale & -0.23 & $-0,21$ & $-0,20$ & $-0,18$ & $-0,28$ & $-0,33$ \\
\hline
\end{tabular}


Spostamenti verticali decennali dei caposaldi costieri

(+ innalzamento, - abbassamento)

\begin{tabular}{|c|c|c|c|c|c|c|}
\hline & $1891-00$ & $1901-10$ & 1911.20 & 1921.30 & $1931-40$ & $1891 \cdot 10$ \\
\hline & $\mathrm{cm}$ & $\mathrm{cm}$ & ('m & $\mathrm{cm}$ & $\mathrm{cm}$ & $\mathrm{em}$ \\
\hline Fennoscandia & +2.8 & $+5,2$ & $+3,9$ & $+6,0$ & $+0,5$ & $+3,7$ \\
\hline Lettonia & $-4,2$ & $-8,0$ & $+0,4$ & $-1,6$ & $\ldots$ & $+-0, \pi$ \\
\hline Germania & $-3,3$ & $+0,3$ & $+1,2$ & $-0,7$ & $+2,1$ & $-0,1$ \\
\hline Olanda & $-2,0$ & $-3,2$ & $-0,6$ & $+1,1$ & +2.8 & $-0,4$ \\
\hline Inghilterra & $+0,1$ & $-2,1$ & - & $-3,0$ & $-\mid-1,4$ & $-1,0$ \\
\hline Francia W & $+1,4$ & $-5,5$ & $+0,9$ & $-1,3$ & $-1,6$ & $-1,2$ \\
\hline Francia $\mathrm{S}$ & $+-0,2$ & $+0, \overline{5}$ & $+0,2$ & $-0,6$ & $-2,6$ & $-0,5$ \\
\hline Spagna & $+1,7$ & $+4,9$ & $-4,2$ & $+3,3$ & - & $+1,4$ \\
\hline Portogallo & - & - & $+1,3$ & $+4,4$ & $+2,5$ & $+3,7$ \\
\hline Azzorre & - & - & $+0,8$ & $-1,6$ & - & $-0,1$ \\
\hline Corsica & - & - & - & $+0,1$ & - & $+-0,1$ \\
\hline Sardegna & - & $-0,9$ & 0 & $-2,1$ & - & $-1,0$ \\
\hline M. Tirreno & $-0,2$ & $-0,3$ & $-1,1$ & $-0,9$ & $+0,6$ & $-0, t$ \\
\hline Sicilia & - & $+0,9$ & $-0,5$ & - & - & $+0,2$ \\
\hline M. Adriatico & $-0,3$ & $-1,4$ & $-0, i$ & -0.6 & $-1,6$ & $-0,9$ \\
\hline Canale Suez & - & - & - & - & $+0,1$ & $\therefore 0,1$ \\
\hline Algeria & $+0,8$ & --0.9 & $+1,5$ & $-1,8$ & $+1,3$ & $-0,1$ \\
\hline Marocco & -- & - & - & - & -0.7 & -0.7 \\
\hline Costa d'Oro & 一 & - & - & 一 & $-3,1$ & $-3,1$ \\
\hline Argentina & - & - & $+1,2$ & $-2,5$ & $-0,2$ & $-0,5$ \\
\hline $\mathrm{USA} \cdot \mathrm{S}$ & - & - & $-2,8$ & $-1,6$ & $-3,6$ & $-2,7$ \\
\hline USA - E & - & $-0,1$ & -2.2 & $+0,5$ & $-3,8$ & $-1,4$ \\
\hline Alaski & - & - & - & $-0,7$ & $+2,1$ & $+0,7$ \\
\hline USA - W & - & -1.6 & $+1,3$ & $-1,4$ & $-1,1$ & $-0, \pi$ \\
\hline Canada E & - & $+0,9$ & $+0,3$ & $+0,4$ & $+0,3$ & $+0,5$ \\
\hline Canada W & - & - & +0.3 & $-0,3$ & - & 0 \\
\hline Balboa &.- & - & $+0,9$ & $-0,4$ & $-2,8$ & $-0,8$ \\
\hline Cristobal & - & - & $+1,7$ & $+2,5$ & $+1,5$ & $+1,9$ \\
\hline Hawai & $\cdots$ & - & $+0,3$ & $+1,0$ & $+1, \tilde{5}$ & +0.9 \\
\hline Nuova Zelanda & - & - & $-2,6$ & $+2,1$ & $+0,3$ & 0 \\
\hline Australia & $+1,4$ & $-2,8$ & $+3,5$ & $+2,1$ & $-0, \pi$ & $+0,7$ \\
\hline Madagasear & - & - & - & $\rightarrow$ & $+0,6$ & $+0,6$ \\
\hline Arabia & $+0,2$ & $+1,6$ & $+0,3$ & - & - & +0.7 \\
\hline India & $+1,4$ & $-0,5$ & 0 & 0 & $+1,0$ & $+0,1$ \\
\hline Burma & $+2,0$ & $-2,1$ & $-1,6$ & $-1-2,4$ & $+3,4$ & $+0,8$ \\
\hline Is. Andamane & $-2,1$ & $-1,4$ & $-0,8$ & - & - & $-1,4$ \\
\hline Indie SE & - & - & - & $-0,8$ & - & $-0,8$ \\
\hline Giappone & - & 0 & $-2,2$ & $-2,5$ & - & $-1,6$ \\
\hline Formosa & - & - & $-1,2$ & $+1,2$ & - & 0 \\
\hline
\end{tabular}


Dall'esame di questa tabellina risulta quanto segue:

1) In tutti i sei casi di divisione della superficie terrestre ora considerati si ha sempre un sollevamento della crosta stessa nelle zone polari ed un ahloassamento nella fascia equatoriale.

2) Il sollevamento medio per le calotte polari è dell'ordine di $0,3 \mathrm{~mm} /$ decennio, l'ahhassamento equatoriale è dello stesso ordine.

3) Il sollevamento massimo ai due poli è di quasi $0,5 \mathrm{~mm} / \mathrm{de}-$ cennio, l'ahliasaamento massimo all'equatore risulta pure di quasi 0,5 mm/decennio.

Conclusioni. - Attualmente la crosta terrestre sulisce una deformazione corrispondente ad una diminuzione dello schiacciamento lungo l'asse polare. Essa tende ad avvicinarsi alla forma sferica. L'aumento del diametro polare risulta di quasi $1 \mathrm{~mm}$ per decennio, dello stessa ordine risulta la diminuzione del diametro equatoriale. Tutto cio potrel,he essere in relazione con la diminuzione della pressione esercitata dai ghiacciai sulle calotte polari in corrispondenza alla loro attuale notevole fase di fusione.

$$
\text { Trieste - Istituto Talassografico - Giugno } 1953 .
$$

\section{RIASSUNTO}

Sulla base dei dati mareografici di tutte le stazioni della Terra si motte in evidenza come attualmente la crosta terrestre si deformi con tendenza a diminuire lo schiacciamento lungo l'asso polare. L'aumento del diametro polare risula di quasi $1 \mathrm{~mm}$ per decennio, la diminuzione dei diametro equatoriale è dello stesso ordine.

\section{SL'MMARY}

On the basis of marinographical data from all the stations of the Earth, it is shown how the terrestrial crust is actually deforming with a tendency to diminish the shortening along the polar axis. The increase in the polar diameter has become about $1 \mathrm{~mm}$ for every ten years, and the decrease in the equatorial diameter is of the same order. 


\section{BIBLIOGRAFIA}

Polli S.: Livello medio del mare nella livellazione di precisione. Boll. Soc. Adr. Sc. Nat., XLII (1946), 52-56, Trieste.

Polli S.: Il graduale aumento del livello marino determinato per 30 porti del Mediterraneo. Boll. Soc. Adr. Sc. Nitt, Trieste, vol. XLII, 1946.

Polli S.: Gli attuali movimenti verticali delle coste italiane. Tecnica Italiana, Trieste, N.S., $4,19.17$.

Poll S.: Registrazioni dei bradisismi costieri. Geofisica pura e applirati, Milano, vol. XII, fase. 3-1, 19.18 .

Polli S.: Misura dei lenti movimenti verticali della superficie terrestre. Riun. Ass. Geofis. Ital., 10-11 giugno 1952.

Polli S.: Gli attuali movimenti verticali delle coste continentali. Annali di Geofisica, vol. V. n. A, 597-602, 1952. 